\title{
Neonatal Intensive Care Unit Awareness Month
}

\section{JaNeen Cross, DSW, MSW, MBA}

The National Perinatal Association (NPA)is an interdisciplinary organization that strives to be a leading voice for perinatal care in the United States. Our diverse membership is comprised of healthcare providers, parents \& caregivers, educators, and service providers, all driven by their desire to give voice to and support babies and families at risk across the country.

Members of the NPA write a regular peer-reviewed column in Neonatology Today.

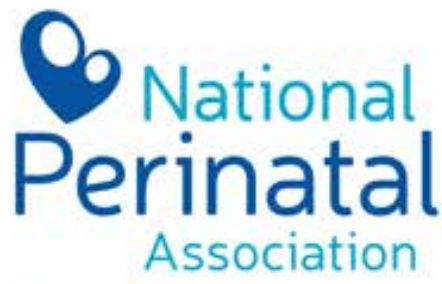

Educate. Advocate. Integrate.

\section{"September is Neonatal Intensive Care Unit}

Awareness Month. As awareness is vital this month, this article will discuss the preliminary findings from a qualitative study to improve awareness about the experiences of women of color in the NICU."
September is Neonatal Intensive Care Unit Awareness Month. As awareness is vital this month, this article will discuss the preliminary findings from a qualitative study to improve awareness about the experiences of women of color in the NICU. Health disparities, social determinants, and concerns for provider bias are key themes in this study. Recommendations are offered to help support the needs of minority parents in the NICU.

\section{"Many women struggle with healing post-delivery while adjusting to the physical, mental, and emotional toll of having their infant in the NICU."}

\section{Health Disparities}

Many women struggle with healing postdelivery while adjusting to the physical, mental, and emotional toll of having their infant in the NICU. In this study, women reported poorly controlled/managed chronic health conditions prior to and during the perinatal period. These mothers identified high blood pressure, diabetes, and prolonged hospitalizations prior to delivery. African Americans are disproportionately affected by diabetes, high blood pressure, and heart disease. (1) These conditions can worsen in pregnancy and jeopardize the health of mother and infant. (1) Struggling with chronic health conditions can contribute to further exhaustion, mental and emotional challenges while providing daily care and breastfeeding to a NICU infant.

\section{Social Determinants}

In this study, the experience of minority mothers in the NICU deviates from most women in that the experience has an implication for life goals. Women in this study talked about how the NICU experiences fostered reflection on their life goals. In this way, they want to improve their circumstances in life and commit to meeting life goals. These goals include establishing independent housing, furthering educational goals, and improving their credit. Many of these women alluded to lessthan-ideal life circumstances and awareness that housing, education, and financial (social determinants) improvements are needed to improve their life. Social determinants provide an accurate understanding of the disparities (1) and the experiences of study participants.

Healthy People 2030 define social determinants of health (SDOH) as environmental conditions where people live, learn, and work that affect outcomes and risks to health, functioning, and quality of life. (2) The five domains of $\mathrm{SDOH}$ include economic stability, education access and quality; health care access and quality; neighborhood environment, social and community context. (2) The areas of the desired improvement expressed by study participants align with the domains for $\mathrm{SDOH}$. Study participants' reaffirmation of these goals stems from a desire to provide a better life to their NICU infant than what they currently experience.

\section{NICU Rollercoaster}

Like the experiences of all mothers with infants in the NICU, women of color highlight the rollercoaster of emotions. Women disclose antithetical feelings when expressing the experience of having an infant in the NICU. These feelings include happy/sad, comfortable/uncomfortable, bond/loneliness, and closeness/rejection. Mothers in a NICU also cite feelings of exhaustion, fear, surprise, and confusion. Study participants identified sadness and worry during the NICU course; however, it is unknown if they experienced or met full criteria for depression, anxiety, stress, or trauma disorders. It is important to note that in the first post-partum year, an estimated $20-30 \%$ of NICU parents have a diagnosable mental disorder. (3) It is recommended that NICU mental health professionals (NMHP) inter-

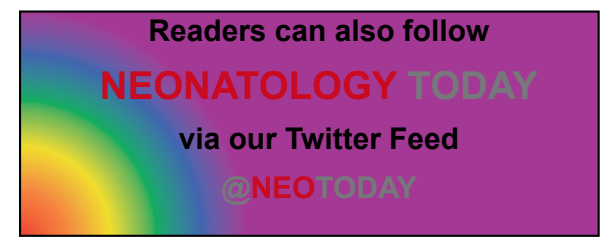


act with all parents to provide screenings for emotional distress, antenatal screenings, layered levels of support, and telemedicine support. (3)

\section{"Although the emotions associated with having an infant in the NICU can vary throughout a NICU stay (days, weeks, or months), two common factors that influence the experiences of minority women are the health status of the infant and the type of interactions with NICU providers."}

Study participants also have very positive experiences in the NICU and express feeling thankful, heightened mood, and amazement with the NICU experience. Although the emotions associated with having an infant in the NICU can vary throughout a NICU stay (days, weeks, or months), two common factors that influence the experiences of minority women are the health status of the infant and the type of interactions with NICU providers.

\section{Provider Bias}

Study participants expressed concerns about trust and worries about the safety of their infant during the NICU course. The concern and worry expressed by study participants are warranted. Race and insurance status increases the potential for implicit bias. (4) When implicit bias occurs, patients receive less attention to medical needs, postponed medical care, and insufficient quality of care. (4) In the NICU, parents should function as healthcare team members, and their feedback incorporated into the treatment plan. (5) NICU providers' communication and teaching activities were pathways for establishing trust and assurance of infant safety for study participants. Participants evaluated their ability to trust the NICU providers through communication, teaching, and education activities.

The study showed a correlation between positive experiences in the NICU, quality, and quantity of provider communication, teaching and education, and perceptions of a welcoming environment. It is widely known that NICU provider communication and parent education are standards of care and essential components in the NICU journey. The quality of provider communication with minority populations can increase their involvement in family-centered care. (6) NICU staff education should focus on methods for involving parents in the care of the infant, enhancing and expanding family-centered developmental care. (6) In addition, NICU staff education needs to include awareness and methods for delivering culturally effective care, which includes diversity (i.e., race, gender, sexual orientation, ethnicity, spirituality, socioeconomic status), cultural traditions, and care preferences. (6)

\section{Conclusion}

It is essential to bring awareness to the experiences of all mothers and families who experience the NICU. Although there are some shared experiences of having an infant in the NICU, unique experiences occur for minority women. There is a burden of care-related social determinants of health, health disparities, implicit (and explicit) bias. These concerns require NICU providers to make concerted efforts to establish therapeutic relationships. NICU providers must prioritize and offer targeted support for biopsychosocial needs and ensure an environment of trust and safety. The latter is stressed as it is sometimes assumed that provider position and title imply trust and safety, where, in the case of minority populations, this role may strain and challenge the provider/parent relationship. Regarding NICU provider awareness, the Interdisciplinary Recommendations for the Psychological Support of NICU Parents provide standards of care and a starting point in bridging the disparity gap.

\section{References:}

1. Cross, J., Brown, D., Groudine, R. (2020). Disparities in maternal and child health among African American Women. Intersection in Practice, 14, 21-23.

2. Department of Health and Human Services (n.d.) Social determinants of health. Retrieved from https://health.gov/ healthypeople/objectives-and-data/social-determinantshealth

3. Hynan, M., Steinberg, Z., Baker, L., Cicco, R., Geller, P., Lassen, S., Milford, C., Mounts, K., Patterson, C., Saxton, S., Segre, L., \& Stuebe, A. Recommendations for mental health professionals in a NICU. Journal of Perinatology, 35, S14-S18.

4. The Joint Commission. (2016). Implicit bias in health care [Issue brief]. https://www.jointcommission.org/resources/ news-and-multimedia/newsletters/newsletters/quick-safety/ quick-safety-issue-23-implicit-bias-in-health-care/

5. Hall, S., Cross, J., Selix, N., Patterson, C., Segre, L., ChuffoSiewert, R., Geller, P., \& Martin, M. (2015).Recommendations for enhancing psychosocial support of NICU parents through staff education and support. Journal of Perinatology, 35, S29-S36.

Disclosure: The National Perinatal Association www.nationalperinatal.org is a $501 \mathrm{c} 3$ organization that provides education and advocacy around issues affecting the health of mothers, babies, and families.

\section{NT}

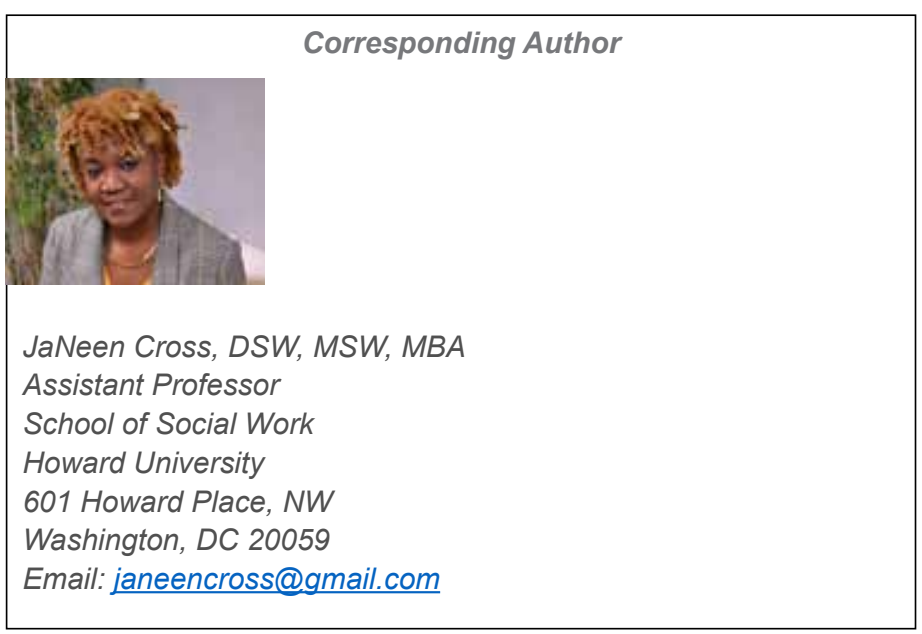

